\title{
A Seamless Handover Mechanism for IEEE 802.16e Broadband Wireless Access`
}

\author{
Kyung-ah Kim ${ }^{1}$, Chong-Kwon Kim ${ }^{2}$, and Tongsok Kim ${ }^{1}$ \\ 1 Marketing \& Technology Lab., KT, Seoul, Republic of Korea \\ \{kka1, tongsok\}@kt.co.kr \\ 2 School of Electrical Engineering and Computer Science, \\ Seoul National University, Seoul, Republic of Korea \\ ckim@popeye.snu.ac.kr
}

\begin{abstract}
Handover is one of the most important factors that may degrade the performance of TCP connections and real-time applications in wireless data networks. We developed a loss-free handover scheme called LPM (Last Packet Marking) for IEEE 802.16e-based broadband wireless access networks. By integrating MAC and network layer handovers efficiently, LPM minimizes the handover delay and eliminates packet losses during handover. Our performance study shows that LPM achieves loss-free packet delivery without packet duplication and increases TCP throughput significantly.
\end{abstract}

\section{Introduction}

At present, existing WLAN has several limitations such as narrow transmission coverage and the interference problem caused by using the ISM (Industrial, Scientific, Medical) band. In order to achieve a higher date rate and wider cell range, the IEEE committee initiated a project 802.16 which standardizes a WBA (Wireless Broadband Access) technologies. The 802.16 project [1] first specified the MAC and physical layers of broadband fixed wireless access system over the 10-66 GHz band. It provides up to several tens of Mbps by using fixed antennas and fixed MSS (Mobile Subscriber Station) in urban and suburban areas. In addition, the IEEE 802.16a 2] modifies the MAC physical layer specifications that facilitates the non-line-of-sight communications over the 2-11 GHz. Furthermore, the baseline specification is now being amended again for mobility enhancement $(60 \mathrm{~km} / \mathrm{h})$ under IEEE 802.16 TGe Mobile Wireless MAN [3], which also deals with transmission power control and power saving.

Compared to wired transmission systems, wireless systems suffer from limited bandwidth and error-prone transmissions. In addition, packet losses and service disruptions may occur during cell HOs (Handovers) in cellular networks.

\footnotetext{
* This work was supported in part by the Brain Korea 21 Project in 2004 and grant No. (R01-2004-000-10372-0) from the Basic Research Program of the Korea Science \& Engineering Foundation.
} 
For the TCP, in particular, lost packets during HO severely degrade the TCP performance because of the sensitivity of the TCP mechanism for packet loss. The TCP retransmits the lost packets and slows down its transmission rate. Even worse, when multiple packets are lost during one congestion window, TCP senders enter into the slow-start phase and decrease the packet sending rate severely.

In this paper, we propose a seamless HO scheme called LPM (Last Packet Marking) for intra-domain mobility in IEEE 802.16e-based broadband wireless packet networks. LPM merges MAC and network layer HO procedures to reduce $\mathrm{HO}$ time and assures a safe $\mathrm{HO}$ by preparing the routing update before the real HO.

This paper is structured as follows: In section 2, we briefly preview the IEEE 802.16 TGe HO procedure and overview the micro-mobility protocols. In section 3, we describe a LPM method for seamless HO on IEEE 802.16e-based wireless broadband access networks. Then, we verify the LPM through computer simulations and present the conclusions in section 5 ,

\section{Background}

\subsection{IEEE 802.16 TGe Handover}

Let us explain the IEEE 802.16 TGe HO procedure briefly. A BS periodically broadcasts a neighbor advertisement management message to identify the network and define the characteristics of the neighbor BS to associated MSS (Mobile Service Station). An MSS may decode this message to find out information about the parameters of the neighbor BS. Each MSS will thus be able to scan the neighbor BS and measure the signal strength. If necessary, an MSS may select neighbor BS and prepare for the future $\mathrm{HO}$ by performing ranging and association procedures. Through ranging, the MSS can acquire the timing, power and frequency adjustment information of the neighbor BS. The target BS-MSS association information is reported to the serving BS.

The MAC layer (L2) HO is divided into two phases; the HO pre-registration phase and the real HO phase. During HO pre-registration, the target BS is selected and pre-registered with the MSS. However, the connection to the currently serving BS is maintained and packets may exchanged during the pre-registration phase. In the real HO, MSS releases the serving BS and re-associates with the target BS.

Now, let's look into the HO procedure in greater detail. Either an MSS or a serving BS may initiate the HO pre-registration. When the MSS initiates the $\mathrm{HO}$ pre-registration, it may indicate a possible target BS from a signal-quality point of view. After the MSS or the serving BS initiates HO pre-registration, the serving BS may acquire information from the neighbor BS regarding their capability of serving the requesting MSS. The serving BS may further notify the neighbor BS (through the backbone) of the impending HO. Fig. 1 shows an example of MAC layer $\mathrm{HO}$ call flow initiated by the MSS $\underline{3}$. After receiving the 


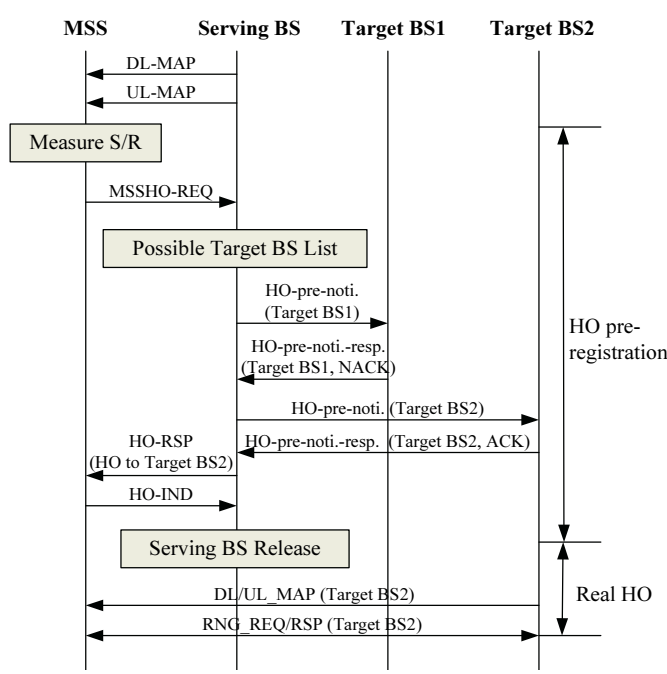

Fig. 1. Example of MAC layer HO call flow initiated by MSS

HO request (MSSHO-REQ) from the MSS, the serving BS sends an HO-prenotification to the candidate target $\mathrm{BSs}$, and the receiving party responds with an HO-pre-notification-response, which include ACK or NACK of impending HO for the MSS. Then, the serving BS selects the target BS and sends an HO-RSP message, including the target BS-ID, to the MSS. The MSS shall transmit an HO-IND message for final indication that it is about to perform a real HO. After the HO pre-registration phase, the real HO procedure is started. The serving BS releases the MSS and the MSS synchronizes with the target BS. Thereafter reauthorization and re-establishment of IP connectivity are taken.

\subsection{Micro Mobility Protocols}

At present, there are many efforts underway to provide Internet services on integrated wireless and wired networks. Supporting an efficient IP mobility is one of the major issues in constructing IP-based wireless access networks. Mobile users will expect the same level of service quality as wired network users. Even though the serving BS of the mobile user changes, IP connections should be continuously maintained transparently. The Mobile Internet Protocol [4] is the current standard for supporting global IP mobility in a simple and scalable manner. However, Mobile IP is targeted for static mobility support where a service continuation is not guaranteed.

A number of solutions like Cellular IP , HAWAII , Hierarchical Mobile IP that support dynamic mobility or cellular networking have been proposed. These approaches aimed to extend Mobile IP rather than to replace it. In order to handle the local movement of mobile hosts without interaction with the MobileIP-enabled Internet, they have adopted a domain-based approach. These intradomain protocols are used for establishing and exchanging the state information 
inside the wireless access networks, so as to get fast and efficient intra-domain mobility or micro-mobility control.

\section{A Seamless Handover Mechanism - LPM}

\subsection{Wireless Access Network Model}

The broadband wireless access network model that we propose uses a routingbased scheme for micro-mobility. The domain is formed by PARs (Packet Access Routers) and BSs (Fig. 2) in a tree structure and is connected to the Internet through the domain root PAR. The BSs use IEEE 802.16e for its wireless interface. For global mobility, the MSS is registered with the address of the gateway PAR on the HA (Home Agent). In the local domain, the MSS is identified by the IP address it uses in its home network. Each PAR maintains the routing cache in a soft-state manner through the periodic routing update information and upward data packets sent by MSSs.

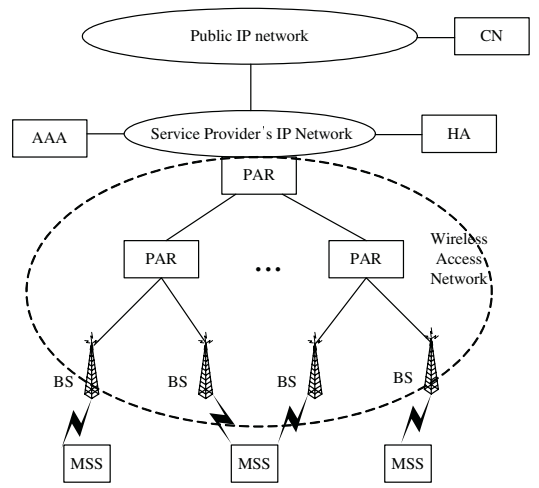

Fig. 2. Wireless Access Network Model

\subsection{Proposed Seamless Hanover Protocol}

In order to provide seamless mobility, the MAC layer (L2) HO and network layer (L3) HO should be integrated to minimize the impact on service performance. If the L3 $\mathrm{HO}$ is started after the L2 $\mathrm{HO}$ has been done, the IP connectivity should be broken for the time being until it is re-established. As a result, packet loss is inevitable. In our scheme, the L3 HO and L2 HO procedure progress concurrently so as to minimize $\mathrm{HO}$ time.

Each BS has BS-ID to BS-IP address mapping table of neighbor BSs in network initiation time. The proposed HO procedure is described in Fig. 3, in which the added procedures to the IEEE 802.16 TGe document are shown in bold and italic. Either the BS or the MSS can initiate HO. Then, the serving BS sends an HO-pre-notification (1) to the candidate target BS. The destination IP 
address of the packet is on the BS-ID to BS-IP address mapping table in the serving BS. The MSS IP address should be added to the original message.

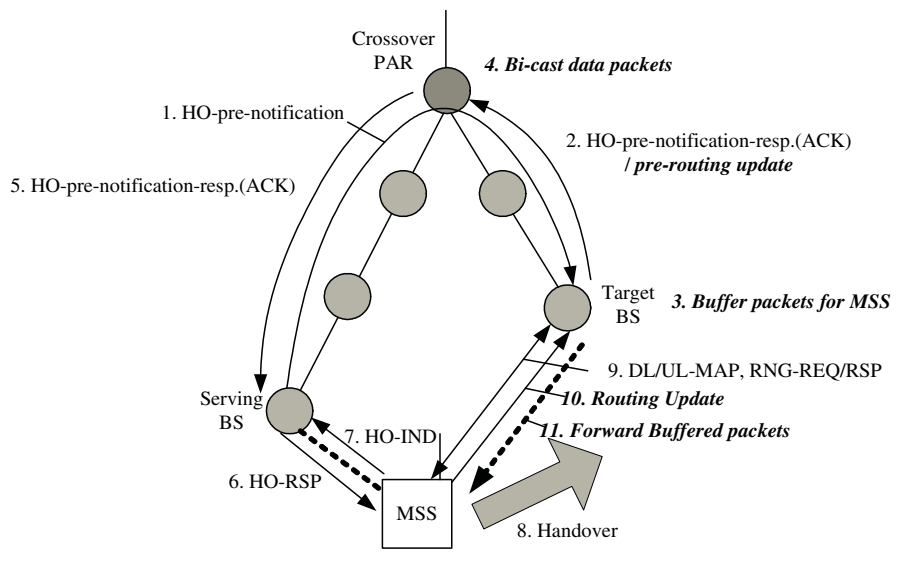

Fig. 3. LPM Handover procedure

When the target BS receives an HO-pre-notification message, it decides whether or not to accept this MSS for HO. Then, it sends an HO-pre-notificationresponse with ACK or NACK to the serving BS. When ACK is the response, the pre-routing update message is sent towards the gateway (2). The sender address of the pre-routing update is the IP address of the impending HO MSS. By prerouting update message, a routing entry in the routing cache of PARs is added in the path through the target BS to cross-over PAR, which is the branching ancestor of the serving $\mathrm{BS}$ and the target BS. Then, the target BS prepares the buffer for the MSS (3), which assures the removal of packet loss during L2 HOs. When the crossover PAR receives the pre-routing update message, it bi-casts the data packets toward the MSS in the direction of both the serving and target BSs (4). The PAR that receives the pre-routing update can know whether it is a crossover or not by looking up the routing cache. If another different routing entry for the MSS is in the cache, then it is a crossover PAR. After the serving BS receives the HO-pre-notification-response (5), it exchanges HO-RSP (6) / HO-IND (7) with the MSS, including the target BS information. Then, the MSS starts the real HO. After the real HO, including ranging and association with the target BS (9), the MSS first sends the routing update message (10) towards the gateway to stop bi-casting of the crossover PAR. Then, the target BS forwards the buffered data for the MSS (11). After that, the MSS can continue its normal packet communication. In the proposed mechanism, the data packets received from the serving BS after the HO-pre-notification-response can also be received through the target BS. This is because the crossover PAR bi-casts the data packets just after receiving the $\mathrm{HO}$-pre-notification-response and pre-routing update. Thus, the HO-pre-notification-response signals the time point, after which the data packets for the MSS are prepared on the target BS buffer. We termed our 
proposal as LPM (Last Packet Marking), since the HO-pre-notification-response indicates that last packet before bi-casting has been received through the serving BS.

In cases where several target BSs send the HO-pre-notification-response with ACK, many crossover PARs bi-cast the data packets. At every bi-casting, just one mapping to the new leaf BS (just one downward link) is attached to the routing cache in the crossover PAR. The final routing tree is a subset tree of the full tree of wireless access networks. That is, in the worst case, where the serving BS sends the HO-pre-notification to all BSs in the access network, all BSs can receive data packets for the MSS after HOs. But after the routing update timeout, only the routing entry on the path that the MSS is attached to remains.

When the MSS postpones the real HO after receiving the HO-pre-notificationresponse, then the bi-casted packets should be received through the serving BS and also through the target BS, which results for the MSS to receive duplicated packets. Thus, the target BS should filter out the duplicated packets. However, the IP layer doesn't know the TCP sequence number. So, when the MSS sends the routing update just after the real $\mathrm{HO}$, the information on the last packet received from the serving $\mathrm{BS}$ before the real $\mathrm{HO}$ can be sent to the target $\mathrm{BS}$. The information is the resulting value of the hash function of (IP Header + Fixed Size IP Payload). When the target BS receives this hash value, it then finds the matched packet in the buffer and only forwards the following packets to the MSS to filter out the duplicated packets.

\section{Simulation}

\subsection{Simulation Details}

We used the micro-mobility extension for the ns-2 network simulator based on version 2.1b6. Since IEEE 802.16e is not yet implemented in ns2, we emulated it using an IEEE 802.11 wireless LAN. When no other MSS are contending for wireless resources, the MSS can stably communicate with the BS like in IEEE 802.16e. HO-pre-notification and response were exchanged between the serving and target BSs.

The simulation topology is shown in Fig. 4. The wireless access network is formed with PAR0-PAR5 and the BSs. The TCP source is CN (Correspondent Node) and the receiver is the MSS. All wired links in the access network are $10 \mathrm{Mb} / \mathrm{s}$ duplex links with a 5-ms delay. The CN and gateway (PAR0) link is set to $10 \mathrm{Mb} / \mathrm{s}$ duplex link with a 50-ms delay. MSS connects to BS using the ns-2 CSMA/CA $2 \mathrm{Mb} / \mathrm{s}$ wireless link model. The link layer $\mathrm{HO}$ delay is set to $15 \mathrm{~ms}$. An MSS starts the TCP connection with CN at time 3 and oscillates between BS1 and BS5 at a constant speed from time 5. The MH stays for about 10 seconds before moving to the next BS. The TCP Tahoe is used for TCP mechanism. The TCP window size is set to 20 and the packet size is 1024 Bytes. 


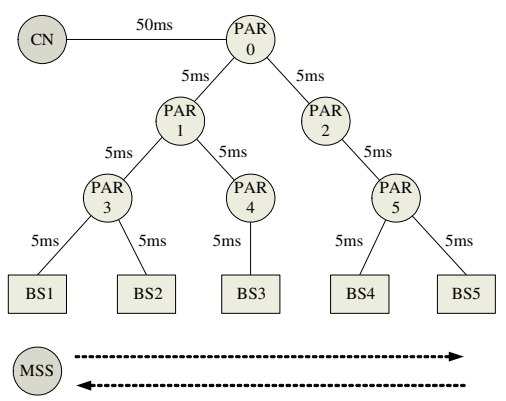

Fig. 4. Simulation Topology

\subsection{Simulation Results}

Figure [5 shows the TCP connection throughput as a function of time. The TCP throughput is measured every 1 second. We call the basic HO scheme as hard $\mathrm{HO}$, in which L3 HO is started after the real HO. All hard HOs have abrupt glitches caused by lost packets. It is well known that a packet loss decreases the TCP performance significantly due to the TCP congestion control. On the other hand, LPM shows no throughput drops on any HO.

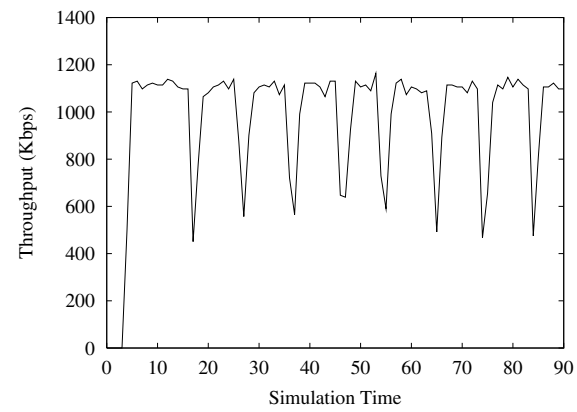

(a) Hard

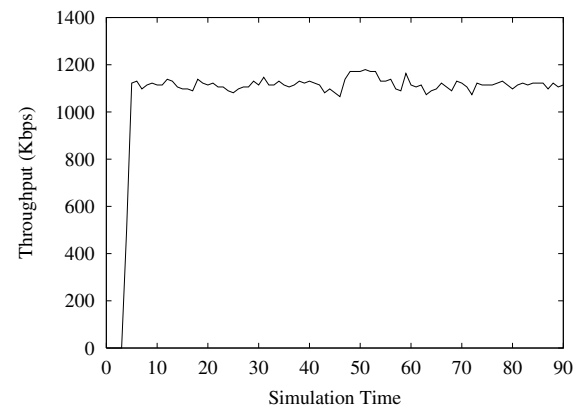

(b) LPM

Fig. 5. TCP throughput

The sender and receiver packet traces of the TCP connection from BS1 to BS2 HO is shown in Fig. 6. All other HO traces showed similar results. In hard $\mathrm{HO}$, the real HO started at time 16.138 and finished at 16.155. Then, the L3 HO (routing update) is done from 16.155 to 16.172 . The network layer HO time is proportional to the round-trip time from BS2 to crossover PAR (PAR3). TCP packet 1714 through 1718 was lost during this period. TCP restarts with slowstart from packet number 1714. But in LPM, no packet loss was observed. The HO-pre-notification message was sent at 16.134 and the response was received at 16.155. Real HO starts at 16.173 and ends at 16.188 . During 16.155 to 16.173 the MSS receives bi-casted packets (1714 and 1718) from the serving BS. After 


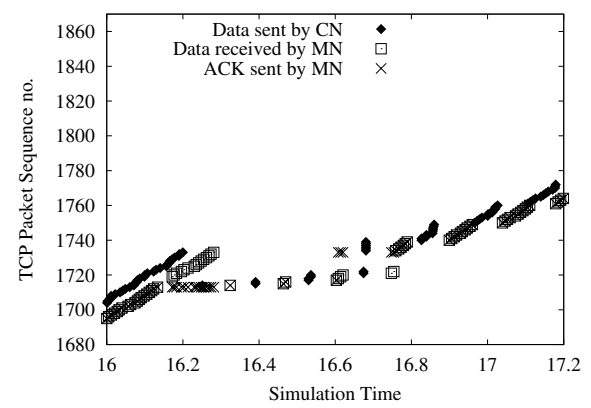

(a) Hard

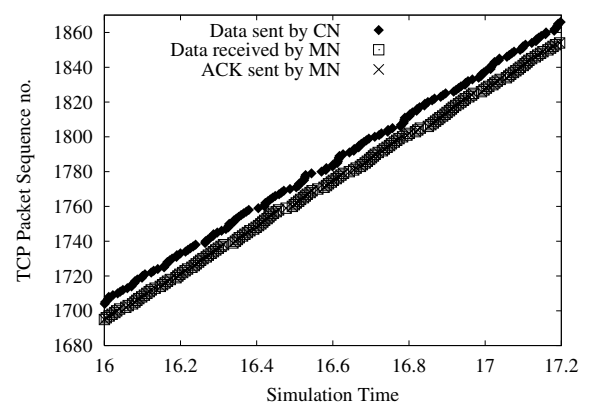

(b) LPM

Fig. 6. Sender and receiver traces of TCP connection

the real HO, the packet from 1714 to 1722 was buffered in the target BS. The target BS filtered out packets below 1719 to remove duplicate packets by hash value, included in the routing update from the MSS. Then, the BS forwarded the packets from 1719 to the MSS.

\section{Conclusions}

We have proposed a new handover scheme called LPM (Last Packet Marking) for micro-mobility in IEEE 802.16e-based broadband wireless packet networks. Through LPM, MAC and network layer handover procedures were done simultaneously to minimize the handover time. We studied the performance of LPM using computer simulation. Our simulation study showed that LPM is free from packet loss and duplication.

\section{References}

1. IEEE Standard 802.16, IEEE Standard for Local and metropolitan area networks, Part 16: Air Interface for Fixed Broadband Wireless Access Systems (2001)

2. IEEE Standard 802.16a, Amendment 2: Medium Access Control Modifications and Additional Physical Layer Specifications for 2-11 GHz (2003)

3. IEEE 802.16 TGe Working Document, (Draft Standard) - Amendment for Physical and Medium Access Control Layers for Combined Fixed and Mobile Operation in Licensed Bands, 802.16e/D4, August (2004)

4. C. Perkins (ed.): IP Mobility Support for IPv4, Internet RFC 3344, Aug. (2002) 